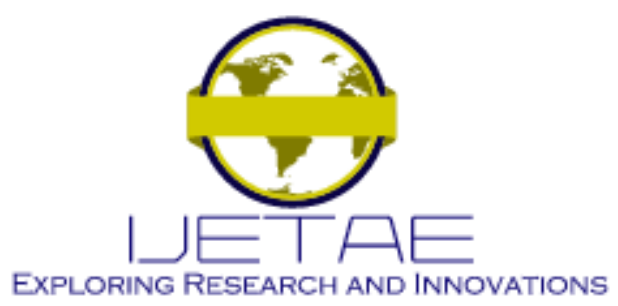

International Journal of Emerging Technology and Advanced Engineering

Website: www.ijetae.com (E-ISSN 2250-2459, Scopus Indexed, ISO 9001:2008 Certified Journal, Volume 11, Issue 11, November 2021)

Manuscript Received: 15 October 2021, Received in Revised form: 06 November 2021, Accepted: 10 November 2021

DOI: 10.46338/ijetae1121_04

\title{
Path Planning for Autonomous Driving of Mobile Robots using Deep Neural Network based Model Predictive Control
}

\author{
Kiwon Yeom \\ Department of Human Intelligence and Robot Engineering, Sangmyung University, South Korea
}

\begin{abstract}
A car-like mobile robot is a nonlinear affine system, and the mobile robot has physical constraints such as velocity and acceleration. Thus, no satisfactory solution may not be provided during self-driving under unknown environments. Although Model Predictive Control (MPC) has provided good performance in terms of control strategy, it is difficult to optimize the control parameters due to the uncertainty and non-linearity of a process. In this paper, the Deep Neural Networks (DNN) based Model Predictive Controller (MPC) is derived for tracking the given path during self-driving. The proposed DNN MPC produces the global optimal solution which has better performance than traditional MPC in terms of the errors of position and orientation. This paper verifies that the proposed DNN MPC based controller can track the desired path with high precision for the car-like mobile robot.
\end{abstract}

Keywords-Path planning, autonomous driving, mobile robot, deep neural network, model predictive control.

\section{INTRODUCTION}

Path planning of the self-driving mobile robot is critical part for autonomous navigation and safety control [1], and the objective is to determine the optimal path without collisions and lane departure during driving from the start position to the goal position.

The generated path for mobile robots must be smooth and applicable to satisfy the characteristics of the mobile robot dynamics.

In general, most path planning algorithms for a car-like mobile robot rely on the information of the surrounding environment. However, mobile robots cannot complete their learning about environment as well as judgment in complex or cluttered environments [2]. Therefore, it requires to develop a path planning method using sparse information of the surrounding environment that mobile robots can quickly adapt to the surrounding environment and autonomously drive to the destination with safety.

As an alternative machine learning method for solving above issue, Deep Neural Networks (DNN) has drawn attention to researchers and there are many applications related to mobile robot's path planning [3].
The DNN method has obvious advantages in path planning and requires less prior information about the environment. Unlike the other supervised learning methods such as regression and back propagation, reinforcement learning does not require much sample data for training process.

Recently, to improve the navigation performance of the autonomous self-driving vehicles, researchers proposed various fusion algorithms [4]. Although the results of the fused strategy are not outstanding, the proposed hybrid algorithms has shown new possibility to advance the ability of the autonomous mobile robot.

From this observation, this paper proposes a new hybrid control algorithm for path tracking of the car-like mobile robot based on Deep Neural Networks based Model Predictive Controller (DNN MPC) as shown in Figure 1, and verifies the effectiveness of the proposed architecture by comparing with other path planning algorithms.

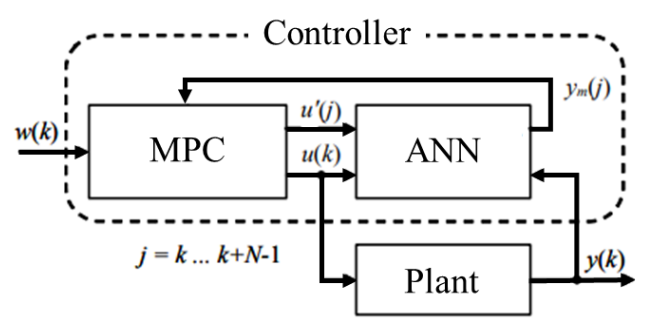

Figure 1. An overview of the DNN MPC controller.

\section{Kinematic Model Of A CAR-Like Mobile Robot}

The path planning considered in this article is based on a car-like mobile robot. In general, the path has different conditions such as straight line, circular arc, turning, etc. The mobile robot can autonomously control the velocity, acceleration and steering angles to successfully achieve the given path tracking. For the purpose of controlling the mobile robot, in this paper a general kinematic bicycle model is applied to the car-like mobile robot. 


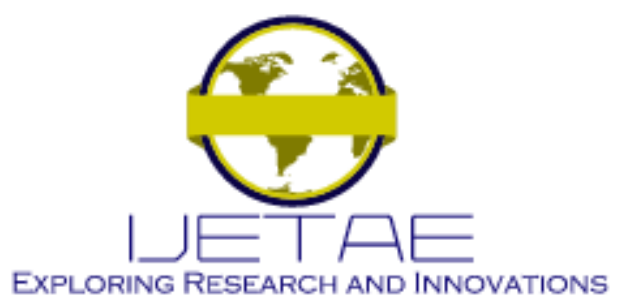

International Journal of Emerging Technology and Advanced Engineering

Website: www.ijetae.com (E-ISSN 2250-2459, Scopus Indexed, ISO 9001:2008 Certified Journal, Volume 11, Issue 11, November 2021)

In this section, the brief explanation for the kinematic model is introduced, and more readings of kinematic bicycle model should be referred in [5].

A four-wheeled vehicle with a fixed rear wheels and front steering wheels can be approximated to the general bicycle model. Therefore, for the purpose of simplicity as in Figure 1. the control mechanism for the car-like mobile robot with the steering handle is analogous to that of the general bicycle.

The bicycle kinematic model, in this paper, is called the front wheel steering model because the front wheel orientation must be controlled to achieve the appropriate direction of the vehicle. On the contrary to this, the rear wheels are linked together through an axle, which is fixed to the vehicle and rotated with no motion of rotation such as roll, pitch and yaw. This study assumes that the wheel rolls without slipping sideways as well. For front-wheel steering of the vehicle, this paper supposes that the plane which the front wheels contact rotates with respect to the vertical axis from the ground as the handle is turned.

The pose of the vehicle is described by the coordinate frame $B$ of which the origin is attached on the center of the rear axle with the forward direction according to the $x$-axis as shown in Figure 2 (left).

In general, autonomous mobile robot's kinematic bicycle model can be represented by the continuous time nonlinear systems and it can be simply described as shown in Figure 2 (right). More detail contents for the kinematic bicycle model can be referred in [6].

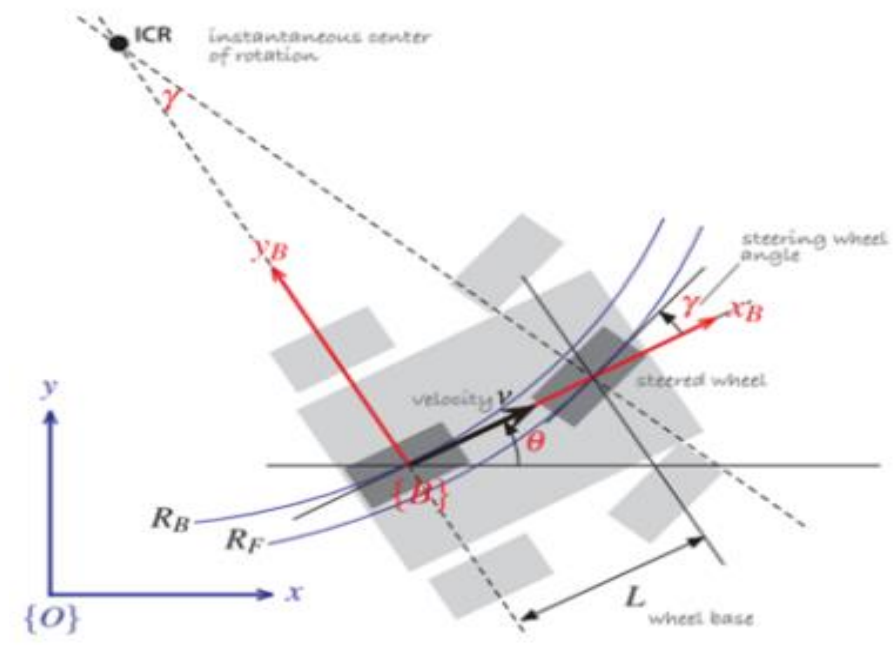

The mobile robot can be described by the generalized coordinates as follows

$$
q=(x, y, \theta) \in C
$$

where $C \subset R^{2} \times S^{1}$. C represents the configuration space of the mobile robot and $S^{1}$ means unit circle with a set of angles $[0,2 \pi)$.

While the mobile robot can change the velocity of the wheels to generate rolling motion, the robot must be able to rotate about a reference point that is a common point perpendicularly lies along the wheel axis. The dashed lines describe the direction along which the wheels cannot move. As shown Figure 1, the lines of no motion are intersected at a point which is known as the ICR (Instantaneous Center of Rotation), and the mobile robot rotates about this point. Thus, the reference point, which is lies on the center of the mobile (see Figure 1. (right)), must follow a circular path as the mobile robot makes a turn. Consequentially, the angular velocity is generated represented as follows

$$
\theta=\frac{D}{R_{B}}
$$

And by simple geometric operation, the turning radius is

$$
R_{B}=L / \tan \gamma
$$

where $L$ is the length between the front and rear wheel bases. This simply means that the turning circle increases as the length is larger. The steering angle $\gamma$ is mechanically restricted, and the maximum value of the steering angle has the minimum value of $R_{B}$.

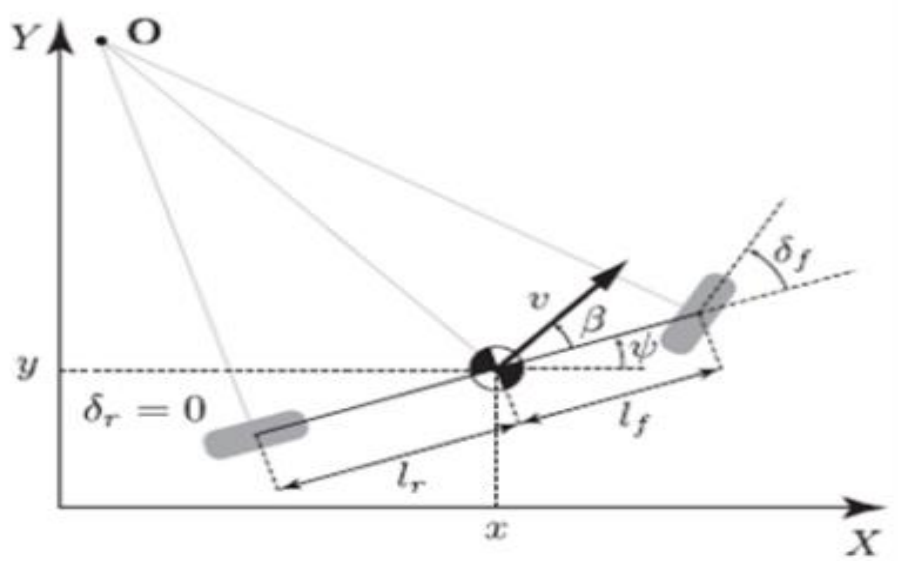

Figure 2. Bicycle model of a car-like mobile robot (figure courtesy of [3]). 


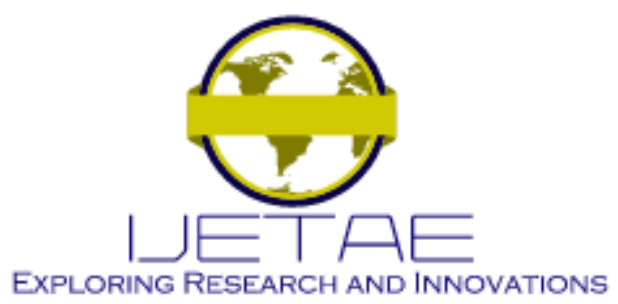

International Journal of Emerging Technology and Advanced Engineering

Website: www.ijetae.com (E-ISSN 2250-2459, Scopus Indexed, ISO 9001:2008 Certified Journal, Volume 11, Issue 11, November 2021)

The lateral dynamics of bicycle model is as follows

$$
\begin{aligned}
& \quad \dot{x}=v \cos (\psi+\beta) \\
& \dot{y}=v \cos (\psi+\beta) \\
& \dot{\psi}=\frac{w}{\mathbb{i}_{r}} \sin \beta \\
& \dot{v}=\alpha \\
& \beta=\tan ^{-1}\left(\frac{\mathbb{1}_{r}}{\mathbb{l}_{f}+l_{r}} \tan \delta_{f}\right)
\end{aligned}
$$

where $x$ and $y$ are the coordinates of the center of mass of the mobile robot which are represented in an inertial frame $(X, Y) . \psi$ is the heading angle of the mobile robot in an inertial frame and $v$ is the speed of the center of mass of the mobile robot. $l_{f}$ describes the distance from the center of the mass to the front, and and $l_{r}$ means the distance from the center of the mass to rear axles.

Compared to other models for autonomous mobile vehicles [7], it is easier to describe the vehicle system on the kinematic bicycle model since there are only two control parameters such as $l_{f}$ and $l_{r}$. In addition, it is possible to design the path planner for the mobile robot with minor modification and build a similar controller for differently sized autonomous mobile vehicles.

$\beta$ is the angle which is made by the velocity vector $v$ and the longitudinal axis of the mobile robot with respect to the center of mass. It is also the same as the angle of the current velocity $v$ of the mobile robot. $\alpha$ is the acceleration of the center of mass of the mobile robot of which the direction is the same as the velocity.

In this lateral dynamics of bicycle model, the major control inputs are considered as the steering angles of the front wheels, namely, $\delta_{f}$ and $\alpha$. Since, in this paper, the mobile robot of which the rear wheels are fixed to the chassis of the vehicle, wherefore the rear wheels cannot be steered, namely $\delta_{r}=0$.

It should be considered in terms of the inertia for more accurate dynamics of the mobile robot. The position coordinates (Eq. 3 and 4) and heading angle (Eq. 5) can be defined in the same as those using the velocity kinematics of the generalized bicycle model (refer [8], for more specific descriptions).
The differential equations for the mobile robot are as follows

$$
\begin{gathered}
\vec{x}=\dot{\psi} \dot{y} \alpha_{x} \\
\vec{y}=-\dot{\psi} \dot{x}+\frac{2}{m}\left(F_{c_{f} f} \cos \delta_{f}+F_{c y}\right) \\
\dot{\psi}=\frac{2}{l_{z}}\left(l_{f} F_{c_{s} f}-l_{y} F_{c y}\right) \\
\dot{X}=\dot{x} \cos \psi-\dot{y} \sin \psi \\
\dot{Y}=\dot{x} \sin \psi+\dot{y} \cos \psi
\end{gathered}
$$

where $\dot{x}$ denotes the longitudinal speed and $\dot{y}$ represents the lateral speed in the body frame of the mobile robot, and $\psi$ describes the ratio of the yaw rotation.

$F_{c, f}$ and $F_{c, r}$ describe the lateral forces of tires for the front wheels and rear wheels, respectively.

For the linear tire model, $F_{c, i}$ is defined as follows

$$
F_{c, i}=-C_{\alpha_{i}} \alpha_{i}
$$

where $\alpha_{\mathrm{i}}$ is the slip angle of the tire $i$, and $C_{\alpha \mathrm{i}}$ is the stiffness of the corresponding tire $i$.

\section{Model Predictive Controller Design}

In Model Predictive Control (MPC) architecture, the control behavior is related to system state and process to satisfy a set of constraints which can be achieved by solving an optimization problem at regular intervals based on sampling time steps. An optimal solution for each control process consists of a certain number of time steps, namely the prediction horizon [9] and [10].

MPC allows to predict and optimize the future behavior of the control system over a finite time horizon, while keeping the future time horizon in account. This can be achieved using the process model which optimize a finite time horizon. The goal is to minimize the objective function (or cost function) of the control system with respect to the future inputs through the predictions [11] and [12].

In general, the target system to be controlled is depicted by First-order or Second-order Ordinary Differential Equation (ODE). Since the control is piecewise constant function, the system can be designed using the differential equation as follows

$$
z(k+1)=f(X(k), u(k))
$$




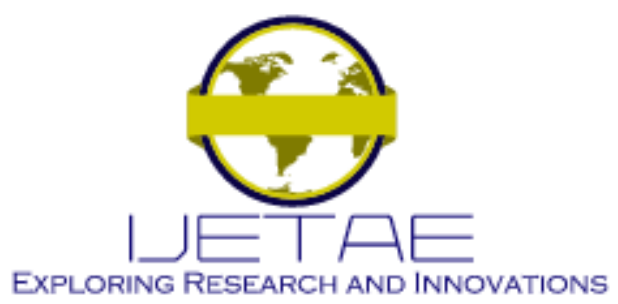

International Journal of Emerging Technology and Advanced Engineering

Website: www.ijetae.com (E-ISSN 2250-2459, Scopus Indexed, ISO 9001:2008 Certified Journal, Volume 11, Issue 11, November 2021)

where $\mathrm{z}(\mathrm{k}+1)$ is the $(\mathrm{k}+1)^{\text {th }}$ output vector, $X(\mathrm{k}) \in R_{z}^{m}$ is the state vector which means the control parameters of the mobile robot such as position and steering angle, namly, $X(\mathrm{k})=[x(\mathrm{k}), y(\mathrm{k}), \theta(\mathrm{k})] . \mathrm{u}(\mathrm{k}) \in R_{u}^{n}$ is the control vector which means the input value of the mobile robot such as velocity and acceleration, namely $u(\mathrm{k})=[v(\mathrm{k})$, $\alpha(\mathrm{k})]^{T}$. This model is used to predict the behavior or find the optimal control input values [13].

As described before, since the MPC is an optimal problem solving for each interval, the mathematical formulation can be described as follows

$$
\begin{gathered}
\min _{u(k)} \Phi(X(k), u(k)) \\
\text { s.t. } \quad z_{0}=z(k) \\
z(k+1)=f(X(k), u(k)) \\
u(k) \in U
\end{gathered}
$$

where $\Phi$ is the objective function of the control system to be minimized, $z_{0}$ is the initial state measured at the current sampling time steps. $z(k+1)$ describes the control behavior using the prediction horizon, and $u(k)$ depicts constraints on the control inputs.

In Eq. 9, the objective function is described as follows

$$
\begin{gathered}
\Phi=\frac{1}{2} \sum_{O_{h \min }}^{O_{\text {max }}}\left\|X_{r}(k+i)-X(k+i)\right\|^{2}+ \\
+\frac{1}{2} \sum_{N_{c h}}^{i=1} \zeta\|\Delta u(k+i)\|^{2}
\end{gathered}
$$

where $O_{\text {hmax }}, O_{\text {hmin }}$ are the maximum and minimum output horizon, respectively. $N_{\mathrm{ch}}$ means the control horizon, and $\Delta u(k+i)$ is an $i^{\text {th }}$ increment such as $\Delta u(k)=u(k+1)-$ $u(k)$.

The objective function with respect to the control horizon $H_{\mathrm{c}}$ results in having the optimized sequence of the control horizon on the entire horizon space such as $u *(k)=$ $\left\{u *(0), u *(1), \ldots, u *\left(H_{\mathrm{c}}-1\right)\right\}$, where $u *(0)$ is the first control for the control plant. More details should be referred [14]. Since this process consequently allows the mobile robot to steer the states toward its reference states, the mobile robot resultingly keeps the path tracking.

\section{DeEP Neural Network BASEd MPC}

Deep neural network used to model the kinematics of the mobile robot is trained according to $z(k)$ and $u(k)$.
Initially, the PID controller controls the car-like mobile robot to track the reference path in order to produce the training data for deep learning. Once the training has been completed, the training data is used for the MPC controller to be adapted during the trajectory tracking process. The overall scheme of deep neural network based MPC is shown in Figure 3.

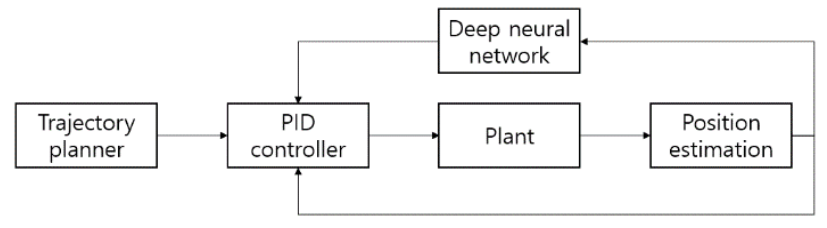

(a) Training setup for the neural network

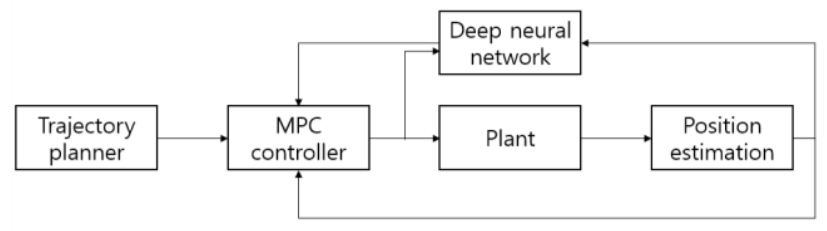

(b) Implementation of DNN MPC model

Figure 3. Scheme of deep neural network based MPC.

In this paper, a deep neural network consists of a fully connected and multilayered. It means that all neurons (or nodes) in previous layer are connected to the neurons (or nodes) in the next layer as shown in in Figure 4.

Since fully connected network becomes agnostic structure, the input data of the neural network is not required any special assumptions. This paper introduces a brief explanation of the architecture for the fully connected deep neural networks, and more readings should be referred in [15] and [16].

The input data of the first layer, namely input layer, consists of the pose $(x, y, \theta)$ and state $(v, \alpha)$. These are the input values of the predicted optimal path at every time horizon. The amount of neurons in the neural network is 160. All data put into the neurons of the input layer are normalized in the range of -1 to 1 . The output from every layer $l[j]$ is generated by applying the activating function $f(\Delta)$ (e.g., hyperbolic tangent sigmoid transfer function) to the inputs from the previous layer with a linear combination manner. The activating function is as follows

$$
l_{j}^{[i]}=f\left(\text { net }_{j}^{[i]}\right)
$$




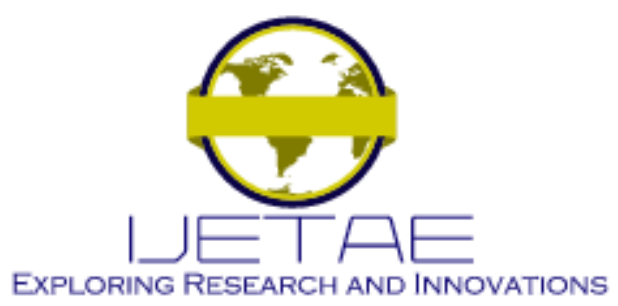

International Journal of Emerging Technology and Advanced Engineering

Website: www.ijetae.com (E-ISSN 2250-2459, Scopus Indexed, ISO 9001:2008 Certified Journal, Volume 11, Issue 11, November 2021)

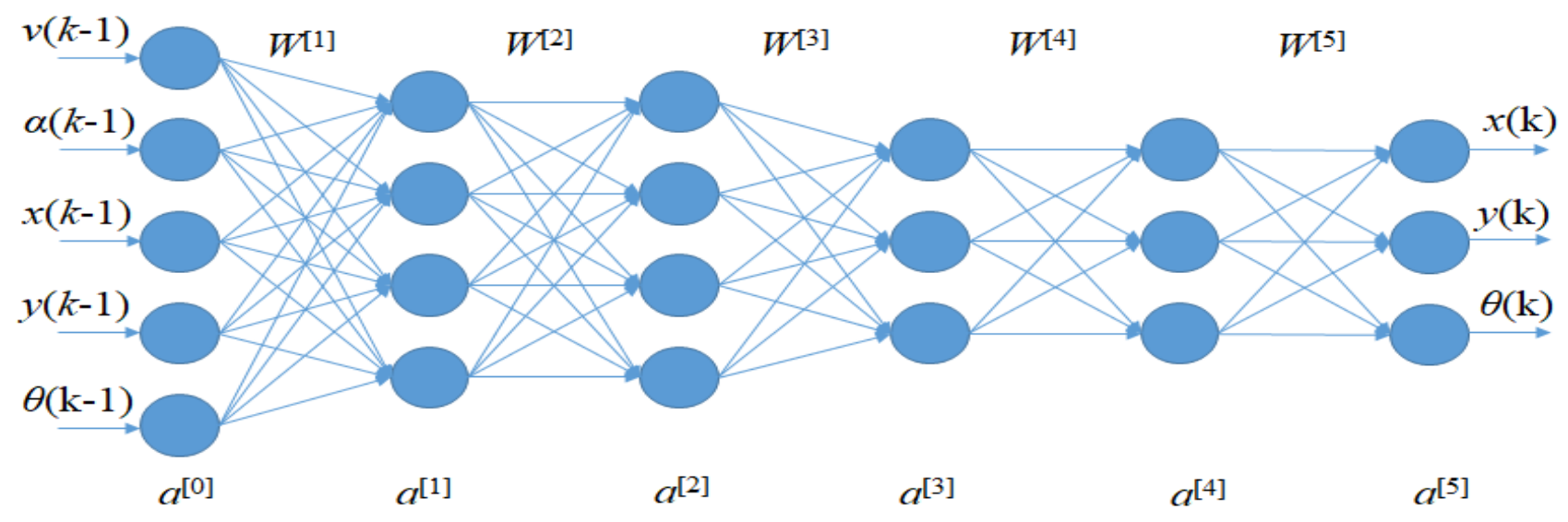

Figure 4. Demonstration of neural networks with four hidden layer

$$
\begin{gathered}
\operatorname{net}_{j}^{[i]}=\sum_{i=1}^{j-1}\left(w_{i j}^{[1]} \cdot l_{j}^{[i-1]}+b_{j}^{[1]}\right) \\
i=1,2, \ldots, p s \quad j=1,2, \ldots, q
\end{gathered}
$$

where $i$ is the total number of neurons in $j^{\text {th }}$ layer, and $j$ is the total number of layers, and $w_{i j}^{[\mathrm{in}]}$ is weights which connect the neurons of the $(j-1)^{\text {th }}$ layer to the neurons of $j^{\text {th }}$ layer, and $b$ is the bias value for neurons of the $j^{\text {th }}$ layer. Based on deep neural networks, the kinematic bicycle model can be represented by

$$
z(k+1)=W_{2} \Phi\left(W_{1} z(k)\right)
$$

where $W_{1}$ is the invariable weight matrix for the training cycle and $W_{2}$ is the updated weight matrix after training. It is updated by a gradient descent algorithm as follows

$$
W_{2}(k+1)=W_{2}(k)-\eta \Phi\left(e^{T}\right)
$$

where $e=\left[x_{\text {est }}(k)-x_{\text {pred }}(k)\right]$ is the error of between the estimated parameters from sensors and the predicted parameters from the neural networks, and $\eta$ is the update rate. More details should be referred [17] and [18].

\section{EXPERIMENTAL ENVIRONMENTS}

A simulator based on the bicycle kinematic model of a car-like mobile robot was developed to validate the DNN MPC method.

As the first stage, the data collection was performed for training the deep neural networks, and it was achieved using the real robot (see Figure 5) which the PID controller was employed to track the path as shown in Figure 6.
The chassis of the mobile robot applied a car-like design with four wheels. Since the axle of the rear wheels is fixed to the body, the rear wheels can only rotate back and forth without pitch, roll and yaw.

The directional control of the front wheels is based on a simplified Ackerman control. In Ackerman steering scheme, the front wheels are enforced to keep the parallel alignment all the time, even though the axle turns on a pivot which is the vertical center position on the steering rod which is connected to a steering servo-motor through a rack and pinion system. The servo-motor is controlled by a microcontroller (Raspberry Pi 3 with Quad Core 1.2GHz) using a pulse-width modulation (PWM) signal and is capable of steering angles of $-45^{\circ} \leq \delta \leq 45^{\circ}$ about front wheels.

In order to generate the reference path $z_{F}(k)$, the current position of the mobile robot is projected onto the street. By comparing the current position and the centerline of the street, the reference path which the mobile robot must follow is generated. The position of mobile robot during tracing the path was estimated and corrected by the Extended Kalman Filter (EKF). An illustration is shown in Figure 5.

The mobile robot to keep tracking the given path is placed ahead of two timeslots (horizon), and the controller steers the car towards the generated reference path trying to minimize the deviation between the current position of the mobile robot and the predicted position on the reference path at any given time. 


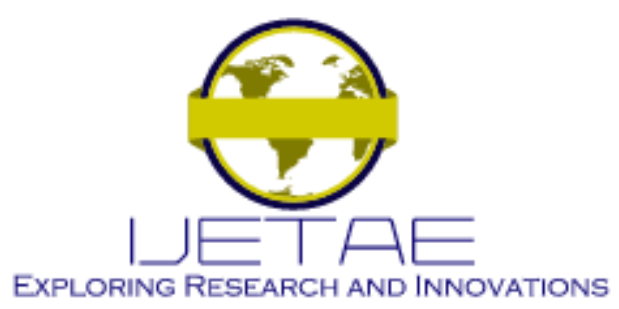

International Journal of Emerging Technology and Advanced Engineering

Website: www.ijetae.com (E-ISSN 2250-2459, Scopus Indexed, ISO 9001:2008 Certified Journal, Volume 11, Issue 11, November 2021)

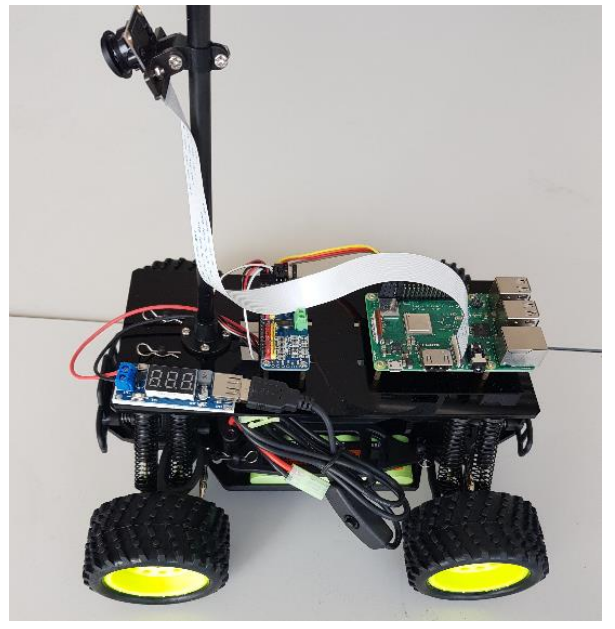

Figure 5. The autonomous car-like mobile robot platform.

The reference path (e.g., the centerline of the lane) for

\section{VALIDATION Of THE DNN BASED MPC}

In this experiment, three different courses were implemented to collect the training data such as straight line and curves such as left and right turn. A path starting from $(0 \mathrm{~mm}, 0 \mathrm{~mm})$ to $(25000 \mathrm{~mm}, 25000 \mathrm{~mm})$ including $\mathrm{pi} / 2$ radian curves is used to validate the proposed DNN MPC control model. The reference path, which the mobile robot should successfully track the given path, was generated by the trajectory planner (see Figure 3).

Firstly, PID controller was used to collect the data for training the neural network.

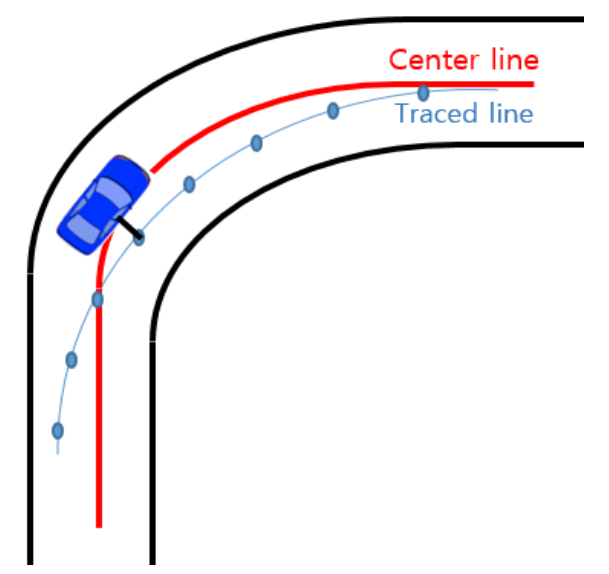

Figure 6. Path tracking scenario and errors illustration.
In Figure 7, 8, and 9 are the results of the DNN MPC controller with respect to the reference path. The results show that the DNN MPC controller is able to successfully approximate to the desired path with very small errors. Of course, since the experimental path is changed with respect to $x$, the errors of $x$ are relatively large in the straight-line part while $y$ and $\theta$ are kept almost constant. However, the proposed DNN MPC controller showed that the mobile robot can successfully track the given path keeping as close as possible to the reference path.

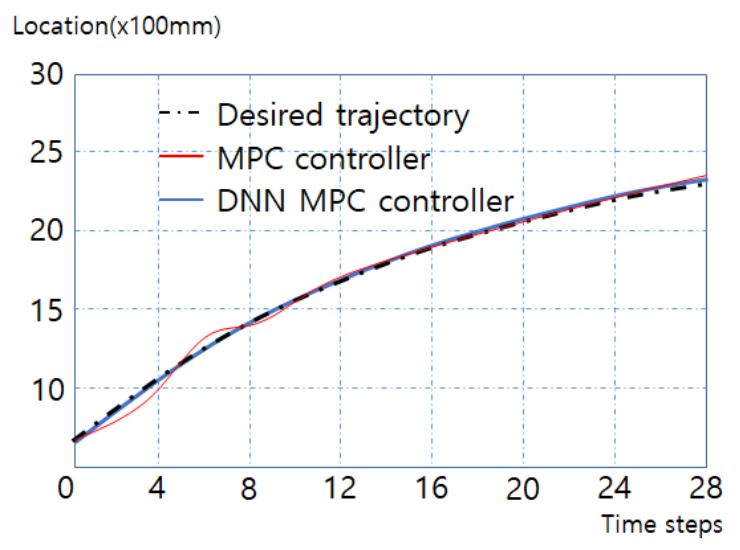

Figure 7. A plot of $x$.

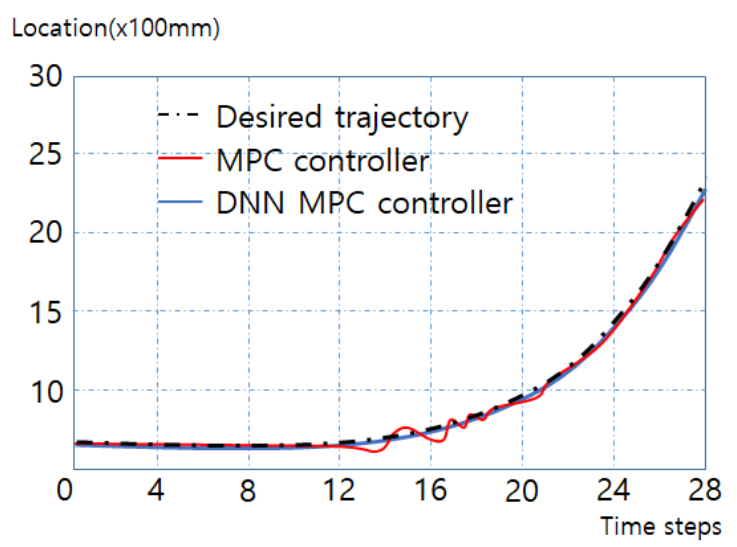

Figure 8. A plot of $y$. 


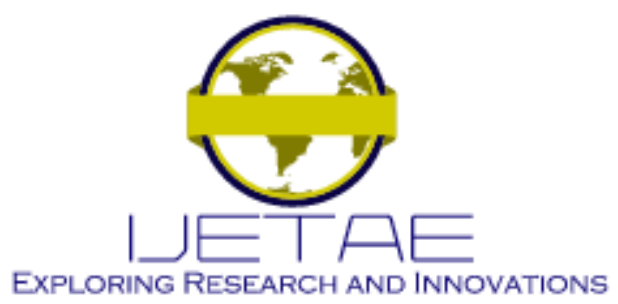

International Journal of Emerging Technology and Advanced Engineering

Website: www.ijetae.com (E-ISSN 2250-2459, Scopus Indexed, ISO 9001:2008 Certified Journal, Volume 11, Issue 11, November 2021)

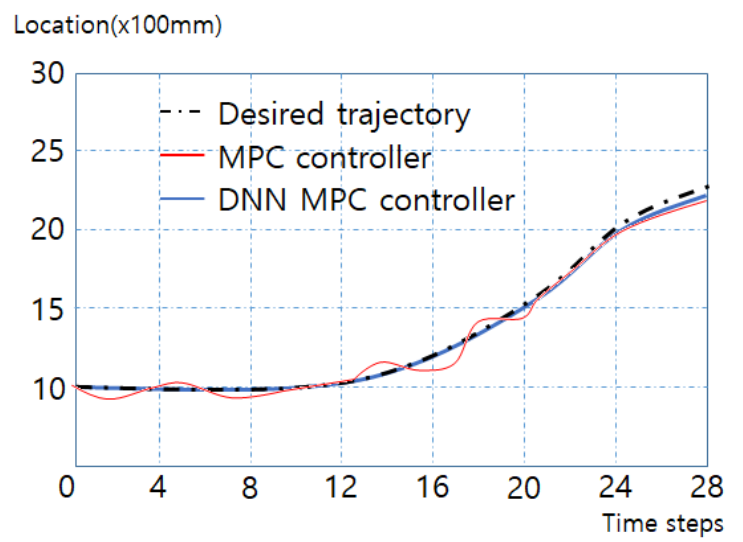

Figure 9. A plot of $\theta$.

However, from the results (see Figure 9), the MPC controller made turn the mobile robot to the left or right with a larger angle than the proposed DNN MPC controller. This made the mobile robot drive a bit away from the desired path than the proposed DNN MPC controller.

In the DNN MPC controller, the highest errors in $x$ and $y$ are $123 \mathrm{~mm}$ and $32 \mathrm{~mm}$ from the desired path, respectively. Contrariwise, in the MPC controller, the highest errors in $x$ and $y$ are $156 \mathrm{~mm}$ and $41 \mathrm{~mm}$.

Table 1 shows the comparison between MPC and the proposed DNN MPC controllers through the average squared error (ASE) with respect to the reference path.

Table 1

Comparison of ASE for MPC and the DNN MPC controller

\begin{tabular}{|l|c|c|}
\hline & MPC & DNN MPC \\
\hline Position $(x, y)$ & 58.08 & 49.96 \\
\hline Orientation $(\theta)$ & 0.385 & 0.286 \\
\hline
\end{tabular}

However, when the mobile robot turns left at a point, all variables $(x, y$ and $\theta$ ) were increased since the mobile robot had to keep tracking the reference path. For the purpose of performance comparisons according to the initial conditions, three different poses were applied with $-5,1$ and 10 streering angles. The MPC and the proposed DNN MPC controllers were applied for tracking performance and the results are shown in Figure 10.

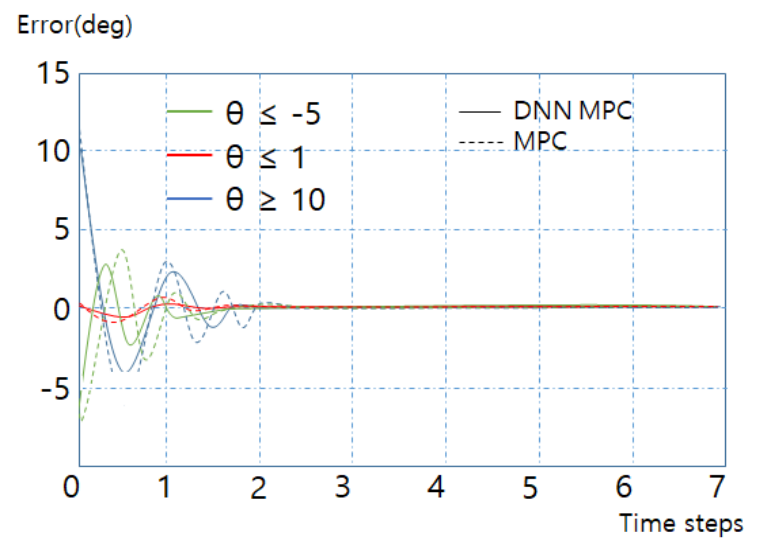

Figure 10. Tracking error of the DNN MPC controller for different conditions.

In Figure 9, there is not much difference in performance of both MPC and DNN MPC controllers. In this experiment, two controllers showed a tendency to a stability within 2.3 seconds. Of course the larger initial steering angles are given, the larger oscillations are observed. However, the system quickly reached the steady state and the oscillations are only observed from the start to around 2 seconds. The largest error of both controllers was less than -6 degrees and both controllers showed the good performance, i.e., the time to reach steady state is around 2 seconds under the largest tilted steering angle conditions.

\section{CONCLUSIONS}

This article introduced a deep neural network based MPC (DNN MPC) controller, which was used for path following of the mobile robot. This paper described the bicycle kinematic model and MPC controller for tracking the mobile robot and designed the framework that combines the MPC controller with the deep neural network.

In this paper the proposed DNN MPC controller was applied to the different environment to follow a reasonable path to demonstrate the feasibility of the DNN MPC algorithm in path tracking.

Experimental results showed that the proposed DNN MPC controller has good performance and make path planning results more practical. 


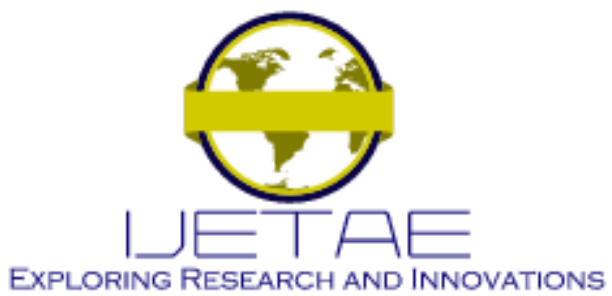

International Journal of Emerging Technology and Advanced Engineering Website: www.ijetae.com (E-ISSN 2250-2459, Scopus Indexed, ISO 9001:2008 Certified Journal, Volume 11, Issue 11, November 2021)

Generally, the DNN MPC controller reduced the tracking errors about $22 \%$ compared to the MPC controller in this article. For the future work, the study of effectiveness of the DNN MPC controller should be performed in more complex environments with other control strategies.

\section{REFERENCES}

[1] N. H. Amer, H. Zamzuri, K. Hudha, and Z. Kadir. "Modelling and control strategies in path tracking control for autonomous ground vehicles: a review of state of the art and challenges", Journal of Advanced Robot and System, vol. 86, pp. 225-254, 2017.

[2] C. Shen, Y. Shi and B. Buckham, "Trajectory tracking control of an autonomous underwater vehicle using Lyapunov-based model predictive control," IEEE Transactions on Industrial Electronics, vol. 65, no 7, pp. 5796-5805, 2017.

[3] X. Yu, W. He, H. Li and J. Sun, "Adaptive Fuzzy Full-State and OutputFeedback Control for Uncertain Robots With Output Constraint", IEEE Transaction on Systems, Man, and Cybernetics, 2020, DOI: $10.1109 /$ TSMC.2019.2963072

[4] A. V. Duka, "Neural network based inverse kinematics solution for trajectory tracking of a robotic arm", Procedia Technol., vol. 12, no. 1, pp. 20-27, Jan. 2014.

[5] K.W, Yeom, "Kinematic and Dynamic Controller Design for Autonomous Driving of Car-like Mobile Robot", International Journal of Mechanical Engineering and Robotics Research, 2018. https://doi.org/10.1155/2018/3039061

[6] K. Takaba, Y, Iiguni, H. Tokumaru, "An improved tracking Kalman filter using a multilayered neural network," Math. Comput. Modell., vol. 23, no. 1-2, pp. 119-128, Jan. 1996.

[7] C. Urmson, J. Anhalt, D. Bagnell, C and Baker, R. Bittner, et al., Autonomous driving in urban environments: Boss and the urban challenge," Journal of Field Robotics, vol. 25, no. 8, pp. 425-466, 2008.

[8] N. H. Amer, H. Zamzuri, K. Hudha, and Z. Kadir, "Modelling and control strategies in path tracking control for autonomous ground vehicles: a review of state of the art and challenges." Journal of Intelligent \& Robotic Systems, vol. 86, no. 2, pp. 225-254, 2017.
[9] C. Liu, S. Lee, S. Varnhagen and H. E. Tseng, "Path planning for autonomous vehicles using model predictive control." IEEE Intelligent Vehicles Symposium, pp. 174-179, 2017.

[10] F. Borrelli, A. Bemporad, and M. Morari, "Predictive control for linear and hybrid systems",http://www.mpc.berkeley.edu/mpccoursematerial, 2015.

[11] A. Carvalho, Y. Gao, A. Gray, H. Tseng, and F. Borrelli, "Predictive control of an autonomous ground vehicle using an iterative linearization approach," Intelligent Transportation Systems, pp. 2335-2340, 2013,

[12] Y. Gao, T. Lin, F. Borrelli, E. Tseng, and D. Hrovat, "Predictive control of autonomous ground vehicles with obstacle avoidance on slippery roads," Dynamic Systems and Control Conference. American Society of Mechanical Engineers, pp. 265-272, 2010.

[13] C. Beal and J. Gerdes, "Model predictive control for vehicle stabilization at the limits of handling," Control Systems Technology, IEEE Transactions on, vol. 21, no. 4, pp. 1258-1269, 2013.

[14] J. Kong, M. Pfeiffer, G. Schildbach, and F. Borrelli, “ Kinematic and dynamic vehicle models for autonomous driving control design." IEEE Intelligent Vehicles Symposium, pp. 1094-1099, 2015.

[15] J. Levinson, J. Askeland, J. Becker, J. Dolson, D. Held, S. Kammel, J. Kolter, D. Langer, O. Pink, V. Pratt, et al., "Towards fully autonomous driving: Systems and algorithms," Intelligent Vehicles Symposium, pp. 163-168, 2011.

[16] A. Carvalho, Y. Gao, S. Lef"evre, and F. Borrelli, "Stochastic predictive control of autonomous vehicles in uncertain environments," International Symposium on Advanced Vehicle Control (AVEC), 2014. C. Peter, "Robot Vision : Second Edition,".

[17] S. Y. Park, C. Y. Lee, "A Study on the forecasting of container volume using neural network," J. Navig. Port Res., vol. 26, no. 2, pp. 183-188 2002. https://doi.org/10.5394/KINPR.2002.26.2.183

[18] B. Karlik, A. V. Olgac, "Performance analysis of Various activation functions in generalized mlp architectures of neural networks, Int. J. Artif. Intell. Expert Syst., vol. 1, no. 4, pp. 111-122. 2011. 